\title{
Vorwort
}

Grundlagenkenntnisse in Chemie sind unerläßlich, um den Aufbau von Organismen und die in ihnen ablaufenden Vorgänge zu verstehen. Dem Studierenden stehen zum Erwerb dieser Kenntnisse mehrere Wege offen: Vorlesungen, Übungen (Praktikum, Seminar) und auch das Selbststudium. Dabei soll das vorliegende Buch eine Hilfe sein. Es ist abgestimmt auf den Gegenstandskatalog für Studierende der Medizin in der Bundesrepublik Deutschland (Fach Chemie); die Neufassung vom Herbst 1976 (2. Auflage 1976) ist berücksichtigt. Geeignet ist das Buch für jeden, der Grundlagenkenntnisse in Chemie erwerben will, vornehmlich für Studierende der Fachrichtungen Medizin, Biologie und Pharmazie sowie anderer Fachrichtungen mit biochemischen Aspekten. In seiner knappen, abbildungsreichen Abfassung soll es zugleich eine rationelle, wiederholende Vorbereitung auf Prïfungen ermöglichen und ein Zusammentragen des Wissensstoffs aus verschiedenen Quellen entbehrlich machen.

Für die Durchsicht von Teilen des Manuskripts danke ich Herm Prof. Dr. Erwin Riedel, Berlin und Herrn Prof. Dr. Walter Ruske, Berlin. Auch dem Verlag de Gruyter sei gedankt für die Bereitwilligkeit, mit der er den Wünschen des Autors entgegengekommen ist.

März 1977

Benno Krieg

\section{Vorwort zur 2.Auflage}

Das Buch ist so gut aufgenommen worden, daß die erste Auflage rasch vergriffen war. Die vorliegende zweite Auflage enthält zahlreiche Verbesserungen. Bei vielen Lesern habe ich mich für kritische Hinweise und wertvolle Anregungen zu bedanken. Mein besonderer Dank gilt den Herren Prof. Dr. Udo Engelhardt, Dr. Jürgen Mittner und Dipl.-Chem. Hans Bauer, Berlin. 